Geometry 85 Topology

Volume 7 (2003) 287-309

Published: 27 April 2003

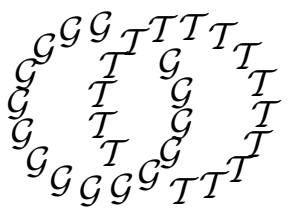

\title{
An algorithm to detect laminar 3-manifolds
}

\author{
IAN AGOL \\ TAO LI \\ Department of Mathematics, University of Illinois at Chicago \\ 322 SEO m/c 249, 851 S. Morgan Street \\ Chicago, IL 60607-7045, USA \\ and \\ Department of Mathematics, 401 Math Sciences \\ Oklahoma State University \\ Stillwater, OK 74078-1058, USA \\ Email: agol@math.uic.edu and tli@math.okstate.edu \\ URL: http://www.math.uic.edu/ ${ }^{\text {agol, }}$ http://www.math.okstate.edu/ ${ }^{\sim}$ tli/
}

\section{Abstract}

We show that there are algorithms to determine if a 3-manifold contains an essential lamination or a Reebless foliation.

\section{AMS Classification numbers Primary: 57M50}

Secondary: 57M25

Keywords: Algorithm, foliation, 3-manifold, lamination

Proposed: David Gabai

Seconded: Cameron Gordon, Joan Birman

(C) Geometry $\& \mathcal{G}$ Topology $\mathcal{P}$ ublications
Received: 13 February 2002

Revised: 18 March 2003 


\section{Introduction}

Essential laminations were introduced by Gabai and Oertel [21], as a generalization of incompressible surfaces [22], measured incompressible laminations [29], Reebless foliations [31], and laminations coming from pseudo-Anosov flows of fibred manifolds [38]. Many properties of Haken manifolds are now known to hold for manifolds containing essential laminations, eg, they satisfy a weak hyperbolization property: either the fundamental group contains $\mathbb{Z}^{2}$, or the manifold has word-hyperbolic fundamental group, as shown by Gabai and Kazez for genuine essential laminations [20], and by Calegari for taut foliations $[8,6,7]$. By the solution of the Seifert conjecture [10,17], if $\mathbb{Z}^{2} \leq \pi_{1}(M)$, then either $M$ contains an embedded incompressible torus, or it is a small Seifert fibered space with infinite fundamental group, for which essential laminations have been classified $[4,1,11,27,30]$. Thus, it is natural to wonder to what extent 3-manifolds with infinite fundamental group and no $\mathbb{Z}^{2}$ subgroup of the fundamental group contain essential laminations.

To resolve this question, it would be useful to have an algorithm to determine whether or not a 3-manifold contains an essential lamination. Jaco and Oertel proved that there is an algorithm to determine if an irreducible 3-manifold is Haken, by showing that if there is an incompressible surface, then there is one among a finite collection of algorithmically constructible normal surfaces. Oertel showed that there is an algorithm to determine whether a manifold contains an affine lamination [32]. Some progress was made by Brittenham, who showed that if a manifold contains an essential lamination, then it contains an essential lamination which is normal with respect to a given triangulation [2]. Brittenham's normalization process was analyzed in depth by Gabai [19], who determined exactly how the normalized lamination differs from the starting lamination. Li gave a criterion on a branched surface embedded in a 3-manifold, called a laminar branched surface, which implies that the manifold has an essential lamination carried by the branched surface, and he proved that any manifold with an essential lamination contains such a laminar branched surface [28]. We show in this paper that the results of Gabai and Li imply the existence of algorithms to determine if a 3-manifold contains an essential lamination or a Reebless foliation, answering Problem 2.3 of [18]. Recently, Roberts, Shareshian, and Stein [33] have given examples of 3-manifolds which admit no Reebless foliation, and Sergio Fenley [12] has extended this to show that their examples contain no essential lamination. The method of proof they use is to show that the groups do not act on order trees. It is unknown whether there are non-laminar 3-manifolds that do not act on order trees, so their approach 
does not provide an algorithm.

The algorithm to determine if a manifold has an essential lamination described in theorem 4.6 proceeds by first finding a finite collection of incompressible Reebless branched surfaces which carry every nowhere dense essential lamination in $M$ which is normal with respect to a particular one-efficient triangulation. Then the algorithm splits into two procedures run in tandem: for each branched surface, one procedure tries to find a laminar branched surface carried by it, which succeeds for at least one branched surface if the manifold contains an essential lamination; the other procedure tries to split each branched surface in all possible ways, which fails for every branched surface in the finite collection if the manifold does not contain an essential lamination.

Acknowledgments We would like to thank Bus Jaco for many helpful conversations about one-efficient triangulations, and Mark Brittenham for finding some errors in an earlier draft of this paper. We also thank the referee for many useful comments, and for noticing some gaps and errors in earlier drafts. The second author is partially supported by NSF grant DMS 0102316.

\section{Laminar branched surfaces}

In this paper, we will assume that all 3-manifolds are orientable, since irreducible non-orientable manifolds are Haken, and therefore laminar.

Definition 2.1 A branched surface $B$ is a union of finitely many compact smooth surfaces glued together to form a compact subspace (of $M$ ) locally modelled on Figure 1(a).

Notation Throughout this paper, we denote the interior of $X$ by $\operatorname{int}(X)$, and denote the number of components of $X$ by $|X|$, for any $X$.

Given a branched surface $B$ embedded in a 3-manifold $M$, we denote by $N(B)$ a regular neighborhood of $B$, as shown in Figure 1(b). One can regard $N(B)$ as an interval bundle over $B$, with a foliation $\mathcal{V}$ by intervals. We denote by $\pi: N(B) \rightarrow B$ the projection that collapses every interval fiber of $\mathcal{V}$ to a point. The branched locus of $B$ is the 1-skeleton of $B$, when viewed as a spine. So, $L$ can be considered as a union of immersed curves in $B$, and we call a point in $L$ a double point of $L$ if any small neighborhood of this point is modelled on Figure 1(a). 


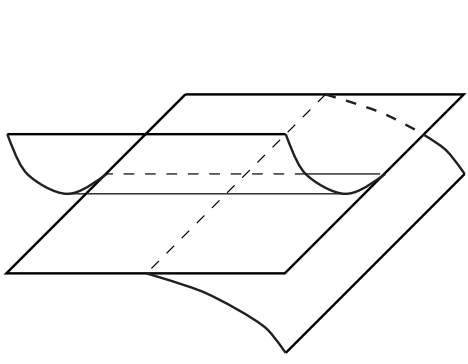

(a)

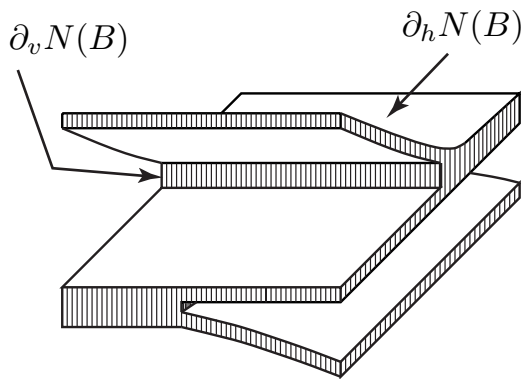

(b)

Figure 1

A (codimension one) lamination $\lambda$ in a 3-manifold $M$ is a foliated, closed subset of $M$, ie, $\lambda$ is covered by a collection of open sets $U$ of the form $\mathbb{R}^{2} \times \mathbb{R}$ such that $\lambda \cap U=\mathbb{R}^{2} \times C$, where $C$ is a closed set in $\mathbb{R}$, and the transition maps preserve the product structures. The coordinate neighborhoods of leaves are of the form $\mathbb{R}^{2} \times x, x \in C$. Let $M_{\lambda}$ be the metric completion of the manifold $M-\lambda$ with the path metric inherited from a metric on $M$. Let $H=\left\{(x, y) \in \mathbb{R}^{2} \mid y \geq 0\right\}$ be the closed upper half plane. An end compression is a proper embedding $d:(H, \partial H) \rightarrow\left(M_{\lambda}, \partial M_{\lambda}\right)$ such that $\left.d\right|_{\partial H}$ does not extend to a proper embedding $d^{\prime}: H \rightarrow \partial M_{\lambda}$.

Given a branched surface $B \subset M$, a lamination $\lambda$ is carried by $B$ if $\lambda \subset N(B)$ and each leaf of $\lambda$ is transverse to $\mathcal{V}$, and fully carries if $\lambda$ meets every fiber of $\mathcal{V}$. Similarly, a branched surface $B^{\prime}$ is carried by $B$ if $B^{\prime} \subset N(B)$ and $B^{\prime}$ is smoothly transverse to $\mathcal{V}$. If $B^{\prime}$ is carried by $B$ and meets every fiber of $\mathcal{V}$, then $B^{\prime}$ is a splitting of $B$.

Definition 2.2 $\lambda$ is an essential lamination in $M$ if it satisfies the following conditions:

(1) The inclusion of leaves of the lamination into $M$ induces a injection on $\pi_{1}$.

(2) $M_{\lambda}$ is irreducible.

(3) $\lambda$ has no sphere leaves.

(4) $\lambda$ has no end compressions.

Theorem 2.3 [21, Proposition 4.5]

(1) Every essential lamination is fully carried by a branched surface with the following properties: 


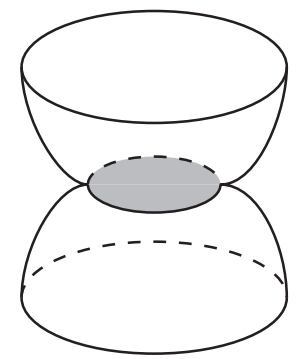

(a)

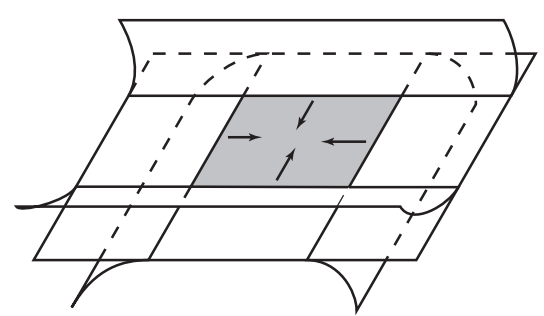

(b)

Figure 2: Sink disks

(a) $\partial_{h} N(B)$ is incompressible in $M-\operatorname{int}(N(B))$, no component of $\partial_{h} N(B)$ is a sphere, and $M-B$ is irreducible.

(b) There is no monogon in $M-\operatorname{int}(N(B))$, ie, no disk $D \subset M-$ $\operatorname{int}(N(B))$ with $\partial D=D \cap N(B)=\alpha \cup \beta$, where $\alpha \subset \partial_{v} N(B)$ is in an interval fiber of $\partial_{v} N(B)$ and $\beta \subset \partial_{h} N(B)$.

(c) There is no Reeb component, ie, $B$ does not carry a sublamination of a Reeb foliation of a solid torus.

(d) $B$ has no disk of contact, ie, no disk $D \subset N(B)$ such that $D$ is transverse to the $I$-fibers $\mathcal{V}$ of $N(B)$ and $\partial D \subset \partial_{v} N(B)$, see Figure 2(a) for an example.

(2) If a branched surface with the properties above fully carries a lamination, then it is an essential lamination.

A branched surface satisfying conditions (a), (b), and (d) of Theorem 2.3 is an incompressible branched surface, and if it satisfies conditions (a)-(d), it is an incompressible Reebless branched surface.

Definition 2.4 Suppose $B$ is an incompressible branched surface. Let $D_{1}$ and $D_{2}$ be two disk components of $\partial_{h} N(B)$, such that $\pi\left(\partial D_{1}\right)=\pi\left(\partial D_{2}\right)$. Then there is a region $K \cong D^{2} \times I$ of $M-\operatorname{int}(N(B))$ such that $D_{1} \cup D_{2}=D^{2} \times \partial I$. If $\pi\left(\operatorname{int}\left(D_{1}\right)\right) \cap \pi\left(\operatorname{int}\left(D_{2}\right)\right)=\emptyset$, then we call $K$ a trivial bubble, and we may collapse $B$ along $K$ by collapsing each $I$-fiber to a point, to get a new branched surface $B^{\prime}$, such that any lamination is carried by $B$ if and only if it is carried by $B^{\prime}$.

Definition 2.5 A sink disk of $B$ is a disk component $D$ of $B-L$ such that $\partial_{h} \pi^{-1}(\bar{D}) \cap \partial_{v} N(B)=\emptyset$ (see figure $2(\mathrm{~b})$ ). 
Definition 2.6 A branched surface $B$ having no trivial bubble in $M$ is called a laminar branched surface if it satisfies conditions $1(\mathrm{a})-(\mathrm{c})$ of theorem 2.3 and has no sink disk.

The following theorem follows from the proof of Lemma 5.4 in [28].

Theorem 2.7 A branched surface carrying an essential lamination which is not a lamination by planes has a splitting which is laminar.

Theorem 2.8 Given a branched surface $B$ which carries no spheres or tori, there is a procedure which terminates if and only if $B$ fully carries a lamination. Moreover, if the procedure terminates, it outputs a certificate that the branched surface fully carries a lamination.

Proof If $B$ is an essential branched surface, then by theorem 2.7, there is a laminar branched surface $B^{\prime}$ fully carried by $B$, ie, $B^{\prime}$ has no sink disks and no trivial bubbles (one need not check the other conditions for laminar branched surface, since $B^{\prime}$ cannot carry a sphere or torus, and $B^{\prime}$ will have no monogon or compression of $\partial_{h} N\left(B^{\prime}\right)$ in $N(B)$ since $B^{\prime}$ is a splitting of $B$, by Lemma 2.5 of [21]). Choose a triangulation $\tau$ of $N(B)$ such that each simplex is transverse to the fibers $\mathcal{V}$ of $N(B)$, and the foliation of the simplex by fibers of $\mathcal{V}$ is linear in the affine structure on the simplex. There is a triangulation of $N(B) \eta$ such that $B^{\prime}$ is embedded in $\eta^{(2)}$. The triangulations $\eta$ and $\tau$ have a common subdivision $\mu$, which carries $B$ in $\mu^{(2)}$, since $\eta$ does. If we choose $\mu$ to be generic, then it will be transverse to $\mathcal{V}$ since $\mathcal{V}$ is linear on each simplex of $\tau$. The procedure proceeds in steps. Let $m=\left|\tau-\tau^{(2)}\right|$ be the number of 3-simplices in $\tau$. Then the $n$th step of the procedure first enumerates all subdivisions $\mu$ of $\tau$ transverse to $\mathcal{V}$ which have $m+n 3$-simplices, up to isotopy transverse to $\mathcal{V}$. For a given $n$, there are only finitely many such subdivisions, which may be found combinatorially, because of the linearity of the foliation $\mathcal{V}$ restricted to each tetrahedron of $\tau$. For each such subdivision $\mu$, it then enumerates all branched surfaces in $\mu^{(2)}$ transverse to $\mathcal{V}$, and checks to see whether they have no sink disk or trivial bubble. The certificate is a description of the subdivision $\mu$ and the branched surface in $\mu^{(2)}$.

Lemma 2.9 For a 3-manifold $M$ with triangulation $\nu$, and a branched surface $B \subset \nu^{(2)}$, there is an algorithm to determine if $B$ is incompressible and Reebless.

Proof We need to check that $B$ satisfies all the conditions of an incompressible Reebless branched surface. The complement of $B$ has a triangulation $\nu^{\prime}$ 
by splitting $\nu$ along $B$. Property 1 (a) of theorem 2.3 states that $\partial_{h} N(B)$ is incompressible in $M-\operatorname{int}(N(B))$, no component of $\partial_{h} N(B)$ is a sphere, and $M-B$ is irreducible. To check that $\partial_{h} N(B)$ is incompressible, we use Haken's algorithm, as improved by Jaco and Tollefson in Algorithm 9.6 of [26], by showing that there is no vertex normal compressing disk for $\partial_{h} N(B)$. One computes the Euler characteristic and number of boundary components of each component of $\partial_{h} N(B)$ in order to check that no component is a sphere. If $M-B$ is reducible, then there would be an essential 2 -sphere in $M-B$ which is a vertex normal surface with respect to $\nu^{\prime}$ by Theorem 5.2 [26]. We may use an algorithm of Rubinstein [34] and Thompson [36] to show that every vertex normal 2-sphere in $M-B$ bounds a ball.

Property 1(b) of theorem 2.3 states that there is no monogon in $M-\operatorname{int}(N(B))$. Again, one need only check that there is no vertex normal disk meeting $\partial_{h} N(B)$ exactly once using the argument in Theorem 6.1 of [26].

Property 1(c) states that there is no Reeb component, ie, $B$ does not carry a torus that bounds a solid torus in $M$. To check this, we compute the normal solution space of surfaces carried by $B$, and compute all vertex surfaces which are tori. For each one, we check that it does not bound a solid torus, by checking that either it bounds no compressing disk using Haken's algorithm, or that it has a compressing disk and bounds a ball with knotted hole, which may be checked using Rubinstein and Thompson's algorithm to recognize the 3-ball $[34,36]$.

Property 1(d) states that there is no disk of contact. We may solve relative normal coordinates to check that no surface carried by $B$ with boundary on $\partial_{v} N(B)$ is a disk, using the method of Floyd and Oertel in Claim 3, page 123 of $[13]$.

\section{Splitting branched surfaces}

Given a branched surface $B \subset M$, let the pared locus $p(B) \subset \partial N_{v}(B)$ be a collection of simple closed curves which are cores for $\partial_{v} N(B)$.

Definition 3.1 A splitting surface $S \subset N(B)$ is a complete $1-1$ immersed surface carried by $N(B)$ such that $\partial S=p(B)$, and such that for each $I$-fiber $J$ of $\mathcal{V}, S \cap J$ is a discrete set. There is a regular open neighborhood $\mathcal{N}(S)$ which fibers over $S$ induced by the fibration $\mathcal{V}$, such that $\mathcal{N}(S) \cap \partial_{v} N(B)=$ $\operatorname{int}\left(\partial_{v} N(B)\right)$ and $\partial \overline{\mathcal{N}(S)}$ is an immersed surface transverse to $\mathcal{V}$. 
Theorem 3.2 A splitting surface $S$ for $B$ determines a unique lamination $\mathcal{L}_{S}$ fully carried by $B$. Conversely, any lamination $\mathcal{L}$ fully carried by $B$ determines a splitting surface $S$, and $\mathcal{L}_{S}$ is a canonical blow-down of $\mathcal{L}$.

Proof For each $I$-fiber $J$ of $\mathcal{V}, J-\mathcal{N}(S)$ is a closed subset. If $J-\mathcal{N}(S)$ has non-empty interior, then consider the quotient $J^{\prime}$ where each open interval of $\operatorname{int}(J-\mathcal{N}(S))$ is identified to a point. Doing this for each $I$-fiber, we get a quotient complex $N(B)^{\prime}$ with fibration $\mathcal{V}^{\prime}$ over the branched surface $B$, where the fibration is by points over the subcomplex of faces of $B$ which do not carry $S$, and is a fibration by intervals over $\pi(S)$. The complement of $\mathcal{N}(S)$ in $N(B)^{\prime}$ is a lamination $\mathcal{L}_{S}$ fully carried by $B$. Moreover, $\mathcal{L}_{S}$ is unique up to normal isotopy.

Conversely, suppose we have a lamination $\mathcal{L}$ fully carried by $B$. Isotope $\mathcal{L}$ so that it meets the endpoints of each fiber of $\mathcal{V}$, possibly collapsing intervals of $\mathcal{V}$ to points over the faces of $B$ which carry $\mathcal{L}$ with multiplicity one. Then the complementary regions which meet $\partial_{v} N(B)$ consist of $I$-bundles over an embedded surface $S$, which we may choose to intersect $\partial_{v} N(B)$ in $p(B)$, so that the region is $\mathcal{N}(S)$ in the topology induced from $M_{\mathcal{L}}$ using the path metric. Thus $S$ is a splitting surface. Consider the intersection with each $I$-fiber $J$, and as before quotient the open intervals of $J-\mathcal{N}(S)$ to points. This quotient map gives a blow-down of $\mathcal{L}$ to $\mathcal{L}_{S}$.

The branched surface $B$ has a cell structure $\tau$. A splitting surface $S$ has an induced cell structure $\tau_{S}$ by pulling back the cell structure $\tau$ under the quotient map $\pi$ (which collapses each fiber of $\mathcal{V}$ to a point).

Definition 3.3 A splitting complex is an embedded complex $c$ in $N(B)$ whose cells are transverse to $\mathcal{V}$ and which has a cell structure $\tau_{c}$ induced by pullback by $\pi$ from $\tau$. Moreover, $c$ is locally embedded in a surface transverse to $\mathcal{V}$, in particular $\operatorname{int}(c)$ is a surface. Let $\partial c$ be the graph of all 1-cells of $\tau_{c}$ which are not incident with two 2-cells of $\tau_{c}$, ie $\partial c=c-\operatorname{int}(c)$. Then we also require that $\partial c \cap \partial_{v} N(B)=p(B)$.

A $B$-isotopy of a splitting complex $c$ is an isotopy of $c$ through splitting complexes which preserves intersections with fibers of $\mathcal{V}$. So a splitting surface $S$ is also a splitting complex, and one should think of a splitting complex as a subcomplex of a splitting surface. If $b \subset c$ is a subcomplex of a splitting complex $c$, then let $B_{1}(b, c)$ consist of the subcomplex of $c$ by adjoining all closed cells incident with $b$ to $b$. This is akin to taking the combinatorial ball of radius 1 
about $b$. Define inductively $B_{k}(b, c)=B_{1}\left(B_{k-1}(b, c), c\right)$. Let the radius of a splitting complex $c$ be $R(c)=\max \left\{k \mid B_{k}(p(B), c) \cap \partial c=p(B)\right\}$. The radius measures the minimal combinatorial distance from $p(B)$ to $\partial c-p(B)$. Suppose that each cell of $B$ has at most $r$ cells (counted with multiplicity) incident with it. Then for any splitting complex $c, B_{k}(p(B), c)$ has at most $r^{k}\left|p(B)^{(0)}\right|$ cells.

Lemma 3.4 There are only finitely many splitting complexes of $B$ with $\leq N$ cells, up to $B$-isotopy, and there is an algorithm to enumerate them.

Proof The $B$-isotopy class of each splitting complex $c$ is determined by the number of cells of each type and how they are glued together along $\pi^{-1}\left(\tau^{(1)}\right)$. To describe a splitting complex with $\leq N$ 2-cells up to $B$-isotopy, we list for each 2-cell $f$ of $\tau$, the number of 2-cells of $c$ which map to $f$ under $\pi$. These 2cells are numbered by the vertical order they come in the $I$-fibration of $\pi^{-1}(f)$. Then we give a pairing of a subset of edges of these cells, and a pairing of some edges with edges of $p(B)$. There are checkable restrictions on the pairings so that the quotient complex is a splitting complex. The pairings must preserve the orderings of the cells, and must not induce branching about $\pi^{-1}\left(\tau^{(0)}\right)$, and there must be exactly one edge paired with each edge of $p(B)$.

Theorem 3.5 There is a sequence of splitting complexes $c_{k}$ of $B$ such that the radius $R\left(c_{k}\right) \rightarrow \infty$ as $k \rightarrow \infty$ if and only if there is a splitting surface $S$ of $B$.

Proof If there is a splitting surface $S$, then $R\left(B_{k}(p(B), S)\right)=k-1 \rightarrow \infty$ as $k \rightarrow \infty$, so we may take $c_{k}=B_{k}(p(B), S)$.

Conversely, given a sequence of splitting complexes $c_{k}$, with $R\left(c_{k}\right) \rightarrow \infty$, we may assume that $\left\{R\left(c_{k}\right)\right\}$ is an increasing sequence. Then for $k \geq K$, the sequence of complexes $\left\{B_{R\left(c_{K}\right)}\left(p(B), c_{k}\right)\right\}$ must have a constant subsequence, since there are at most $\left|p(B)^{(0)}\right| r^{R\left(c_{K}\right)}$ cells in these complexes, and thus there are only finitely many complexes up to $B$-isotopy, by lemma 3.4. Thus, we may find a subsequence $\left\{c_{k_{i}}\right\}$ such that for all $j>i, B_{R\left(c_{k_{i}}\right)}\left(p(B), c_{k_{i}}\right)$ is $B$ isotopic to $B_{R\left(c_{k_{i}}\right)}\left(p(B), c_{k_{j}}\right)$. We may $B$-isotope $B_{R\left(c_{k_{j}}\right)}\left(p(B), c_{k_{j}}\right)$ so that $B_{R\left(c_{k_{i}}\right)}\left(p(B), c_{k_{i}}\right)=B_{R\left(c_{k_{i}}\right)}\left(p(B), c_{k_{j}}\right)$. Moreover, we may choose a small $I$ bundle neighborhood of each $\left\{B_{R\left(c_{k_{i}}\right)}\left(p(B), c_{k_{i}}\right)\right\}$ so that the later stages remain disjoint from it. In the limit, the union of $B_{R\left(c_{k_{i}}\right)}\left(p(B), c_{k_{i}}\right)$ forms a splitting surface $S$.

Theorem 3.6 Given a branched surface $B$, there is a procedure which terminates if and only if $B$ does not carry a lamination. 
Remark The referee points out that this theorem was already known to Oertel.

Proof The algorithm proceeds by enumerating all splitting complexes $c$ using $\leq\left|p(B)^{(0)}\right| r^{N}$ cells, by the algorithm described in lemma 3.4 , for $N=1,2,3, \ldots$. For each $N$, one finds whether there is such a $c$ with $R(c) \geq N-1$, which is algorithmic since given $c$ there is a simple algorithm to compute $R(c)$. If no such complex $c$ exists, then $B$ carries no lamination fully, since if there were a splitting surface $S$, then $B_{N}(p(B), S)$ would be a splitting complex with at most $\leq\left|p(B)^{(0)}\right| r^{N}$ cells and with radius $\geq N-1$.

\section{Constructing essential branched surfaces}

Suppose we split a certain branched surface $B$ along a splitting surface described before. The inverse limit of an infinite splitting is a lamination carried by $B$ by Lemma 4.2 of [21]. Thus, the process of such splitting will stop unless $B$ fully carries a lamination. By Theorem 1 of [21], if an incompressible Reebless branched surface fully carries a lamination, then it is an essential lamination. Therefore, in order to make the algorithm work, we need to find finitely many incompressible Reebless branched surfaces such that one of them fully carries a lamination if $M$ is laminar.

We assume that $M$ is irreducible and has a triangulation $\tau$. We may also assume that $M$ is atoroidal, otherwise $M$ contains an essential torus, and thus has an essential lamination. By [25], there is an algorithm to find an efficient triangulation $\tau$ of $M$, which has the property that the only normal 2-sphere is the vertex linking 2-sphere, and there are only finitely many normal tori (each bounding a solid torus). It has been shown in $[2,19]$ that if $M$ contains an essential lamination, then $M$ contains a normal essential lamination with respect to any given triangulation. By putting normal disks together, as on p. 122 of [13], we can construct finitely many branched surfaces such that any normal lamination is fully carried by one of them. In fact, for any normal essential lamination, by identifying all the normal disks of the same type to one normal disk, we get a branched surface $B$ that is among the finitely many branched surfaces constructed above and $B$ fully carries the essential lamination. By solving certain systems of branch equations, we can algorithmically find all minimal weight disks of contact in $B$ and split $B$ to eliminate all of these disks of contact. This can be done in finitely many steps. The argument of Claims 1-3, pages 122-123 of [13] generalizes from incompressible surfaces 
to laminations to show that, once we have split along all minimal weight disks of contact, we will have a branched surface carrying a lamination isotopic to $\lambda$ which does not carry any 2 -sphere, since $\tau$ was assumed to be an efficient triangulation.

We say that a surface $S$ is carried by $N(B)$ if $S$ is transverse to the $I$-fibers of $N(B)$. In this paper, we always assume our surface $S$ above is embedded in $N(B)$. We define the weight of a surface $S$ to be the number of intersection points of $S$ with the 1-skeleton of the triangulation, and define the length of an arc to be the number of intersection points of this arc with the 2-skeleton. Let $p_{0}$ and $p_{1}$ be surfaces or $\operatorname{arcs}$ in $N(B)$ transverse to the $I$-fibers of $N(B)$. We say $p_{0}$ and $p_{1}$ are $B$-parallel if there is an isotopy $H: F \times[0,1] \rightarrow N(B)$ such that $H(F \times i)=p_{i}(i=0,1)$ and $H(q \times[0,1])$ is a subarc of an $I$-fiber of $N(B)$ for any $q \in F$. We also call this isotopy a $B$-isotopy.

Lemma 4.1 Suppose $B$ fully carries a nowhere dense essential lamination $\lambda$ and $B$ contains no disk of contact and carries no 2-spheres. Let $T$ be a torus carried by $N(B)$ and bounding a solid torus $V$ in $M$. Then, after $K$ steps of splitting, where $K$ depends on $B$ and $T$ (not on $\lambda$ ), $B$ can be split into a branched surface that still carries $\lambda$ (up to isotopy) but does not carry $T$.

Proof If there are subarcs of $I$-fibers of $N(B)$ properly embedded in $M-$ $\operatorname{int}(V)$, then the union of these subarcs is an $I$-bundle over a compact surface whose horizontal boundary lies in $T$. Let $H \subset T$ be the horizontal boundary of this $I$-bundle. Note that we can assume $H$ consists of planar surfaces, otherwise, since $M$ is irreducible, $M$ must be the union of $V$ and a twisted $I$-bundle over a Klein bottle, which contradicts that $M$ contains an essential lamination (because such 3-manifolds are double covered by lens spaces). Then, by splitting along a union of surfaces (in this $I$-bundle) with total weight bounded by the weight of $H$, we get a branched surface that still carries $T$ and $\lambda$ but there is no subarc of any $I$-fiber properly embedded in $M-i n t(V)$.

So we may assume that there is no subarc of any $I$-fiber of $N(B)$ properly embedded in $M-\operatorname{int}(V)$. In the discussion below, we always assume $T$ intersects $\lambda$ transversely. We can perform $B$-isotopy on parts of $\lambda$ pushing $\lambda$ into $V$ as much as one can in the sense that after the $B$-isotopy, for any point $x \in T \cap \lambda$, there is an $\operatorname{arc} \alpha \subset \lambda-\operatorname{int}(V)$ such that, $x \in \alpha, \alpha$ is not $B$-parallel to any arc in $T$, and the combinatorial length of $\alpha$ is bounded by $K_{1}$, where $K_{1}$ depends on $B$ and $T$ (not on $\lambda$ ).

If $\lambda \cap T$ contains circles homotopically trivial in $T$, then as in [1], there are trivial circles bounding disks in $V \cap \lambda$ or $\lambda-i n t(V)$. Then, after some standard 
cutting and pasting on $\lambda$, we can eliminate the trivial circles in $T \cap \lambda$. Note that one can perform such cutting and pasting on laminations because of the Reeb stability theorem (see the proof of Lemma 2.1 of [1]). Moreover, since $N(B)$ does not carry any 2 -sphere, after such cutting and pasting, the lamination is still transverse to the $I$-fibers of $N(B)$. After this operation, by Lemma 2.1 and Theorem 3.1 in [1], $V \cap \lambda$ is either a union of meridional disks or contains a sublamination by annuli (with at most one Möbius band). Moreover, if the second case happens, any non annulus (or Möbius band) leaf in $V \cap \lambda$ is noncompact and simply connected, and each annulus leaf is $\pi_{1}$-injective in $V$.

Suppose $V \cap \lambda$ is a union of meridional disks. In general, a meridional disk (with fixed boundary) can wrap around $T$ many times similar to a Reeb foliation. However, we can perform an isotopy (in fact a Dehn twist) near $T$ to unwrap these disks, see Figure 6.1 in [19] for a schematic picture. Thus, after this unwrapping, there must exist a point $x$ in $T \cap \lambda$ and an arc $\beta \subset V \cap \lambda$ such that $x \in \beta, \beta$ is not $B$-parallel to any arc in $T$, and the length of $\beta$ is bounded by $K_{2}$, where $K_{2}$ depends on $V \cap N(B)$ (not on $\lambda$ ). By connecting $\beta$ and $\alpha$ above together, we get an arc (puncturing through $T$ ) with length less than $K_{1}+K_{2}$. Hence, by splitting $B$ along some disk with diameter less than $K_{1}+K_{2}$, one gets a branched surface that carries a lamination isotopic to $\lambda$ but does not carry $T$.

Now, we suppose $V \cap \lambda$ has a sublamination by $\pi_{1}$-injective annuli (with at most one Möbius band). The annular leaves that are $B$-parallel to subannuli in $T$ (fixing the boundary) form a sublamination of $V \cap \lambda$. After some $B$-isotopy, we can push these annuli (and the simply connected leaves in between) out of $V$ (this can also be done by performing some cutting and pasting on annuli in $T$ ). Such a $B$-isotopy may create some new intersection points in $T \cap \lambda$ and these new intersection points lie in the simply connected leaves of $V \cap \lambda$. After perturbing the simply connected leaves in $V \cap \lambda$, for each new intersection point $x$ in $T \cap \lambda$ (created during the $B$-isotopy above), there is an arc $\alpha_{x} \subset \lambda$ connecting $x$ to an intersection point that is fixed during the $B$-isotopy above. Moreover, $\alpha_{x}$ is $B$-parallel to an arc in an annulus leaf that is pushed out of $V$, and the length of $\alpha_{x}$ is bounded by a number depending only on $T$. So, for each point $x$ in $T \cap \lambda$ after this $B$-isotopy, there is still an arc $\alpha$ with length bounded by a number $K_{1}$ as above, containing $x$, and not $B$-parallel to any $\operatorname{arc}$ in $T$.

So, we can assume $T \cap \lambda$ contains no annulus leaf that is $B$-parallel to a subannulus in $T$, and $T \cap \lambda$ is not a union of meridional disks. In general, an annulus leaf in $T \cap \lambda$ (with fixed boundary) can form a monogon $\times S^{1}$ and wrap around $T$ many times. However, after a Dehn twist near $T$, any annulus 
is isotopic (fixing the boundary) to one with smaller weight, as in the case of meridional disks above. In particular, after this isotopy, there exists a point $x$ in the boundary of this annulus leaf $A$ and an arc $\beta \subset A$ with $x \in \partial \beta$ such that $\beta$ is not $B$-parallel to any arc in $T$ and the length of $\beta$ is bounded by $K_{2}$, where $K_{2}$ is as above. Then, as before, by connecting $\alpha$ and $\beta$, we get an arc (puncturing through $T$ ) with length less than $K_{1}+K_{2}$. Hence, by splitting $B$ along some disk with diameter less than $K_{1}+K_{2}$, one gets a branched surface carrying $\lambda$ but not $T$.

Therefore, in any of the cases, after splitting along a union of surfaces with bounded total weight, we can get a branched surface that carries $\lambda$ (up to isotopy) but does not carry $T$. Since the total weight is bounded by a number that does not depend on $\lambda$, we can enumerate all possible surfaces along which we perform splitting as above, and after splitting we get finitely many branched surfaces from $B$, one of which carries $\lambda$ but does not carry $T$.

Note that after the splitting performed in the proof of Lemma 4.1, new disks of contact may appear, but we can always find and eliminate them by another splitting and taking sub-branched surfaces.

Proposition 4.2 Suppose $B$ fully carries an essential lamination and $B$ contains no disk of contact. Let $C$ be a component of $M-\operatorname{int}(N(B))$ and suppose $C$ contains a monogon. Then, $C$ must be a solid torus in the form of $D \times S^{1}$, where $D$ is a monogon.

Proof Since $B$ fully carries an essential lamination and $B$ contains no disk of contact, $\partial_{h} N(B)$ is incompressible in $M$. Let $D$ be a monogon in $C$, ie, the disk $D$ is properly embedded in $C, \partial D$ consists of two $\operatorname{arcs} \alpha \subset \partial_{v} N(B)$ and $\beta \subset \partial_{h} N(B)$, and $\alpha$ is a vertical arc in $\partial_{v} N(B)$. Let $v$ be the component of $\partial_{v} N(B)$ containing $\alpha$ and $N(v \cup D)$ be a small regular neighborhood of $v \cup D$ in $M$. Then, the intersection of $N(v \cup D)$ and the component of $\partial_{h} N(B)$ containing $\beta$ is a circle $\gamma$. By the construction, $\gamma$ is a trivial curve in $N(v \cup D)$. Since $\partial_{h} N(B)$ is incompressible, $\gamma$ must bound a disk in $\partial_{h} N(B)$. Thus, the component of $\partial_{h} N(B)$ containing $\beta$ is an annulus, $\partial C$ is a compressible torus, and $D$ is a compressing disk for $\partial C$. Since $C$ is irreducible, $C$ must be a solid torus in the form of $D \times S^{1}$, where $D$ is the monogon above.

Suppose $A$ is an annulus carried by $N(B)$. We say $A$ is a splitting annulus if $\partial A$ lies in distinct components of $\partial_{v} N(B)$. 
Proposition 4.3 Let $B$ be a branched surface fully carrying an essential lamination $\lambda$, and suppose $B$ has a monogon. Then, there is a splitting annulus $A$ in $N(B)$ such that $A \cap \lambda=\emptyset$.

Proof We can suppose $B$ does not have any disk of contact. Let $C$ be a component of $M-\operatorname{int}(N(B))$ containing a monogon $D$. Suppose $\partial D=\alpha \cup \beta$, where $\alpha$ is a vertical arc in $\partial_{v} N(B)$ and $\beta \subset \partial_{h} N(B)$. By Proposition 4.2, $C=$ $D \times S^{1}$. Let $\nu=\alpha \times S^{1}$ be the corresponding component of $\partial_{v} N(B)$. By the end-incompressibility of the essential lamination, we can split $N(B)$ along $\lambda$ (by drilling a tunnel) and connect $C$ to another component $W$ of $M-\operatorname{int}(N(B))$, ie, there is a vertical rectangle $R$ in $N(B)$ connecting $\nu$ and another component of $\partial_{v} N(B)$ in $\partial W$ such that $R \cap \lambda=\emptyset$. Note that if $R$ connects $\nu$ to itself, one gets a compressing disk that is the union of $R$ and two monogons, which contradicts that $\lambda$ is essential unless $R$ is parallel to $\nu$. So, existence of such a vertical rectangle $R$ is guaranteed by the end-incompressibility of $\lambda$. After removing a small neighborhood of $R, N(B)$ becomes $N\left(B^{\prime}\right)$ which is a fibered neighborhood of another branched surface $B^{\prime}$ carrying $\lambda$, and now $C$ and $W$ (connected through $R$ ) become a component $C^{\prime}$ of $M-\operatorname{int}\left(N\left(B^{\prime}\right)\right)$, and $\nu$ becomes $\nu^{\prime}$ which is a component of $\partial_{v} N\left(B^{\prime}\right)$. Moreover, since $\lambda$ is endincompressible, we can assume that $W$ is not a $D^{2} \times I$ region.

Since $C=D \times S^{1}$, after this splitting above, $C^{\prime}$ still contains a monogon. As $W$ is not a $D^{2} \times I$ region, $C^{\prime}$ cannot be in the form of monogon $\times S^{1}$. Therefore, by Proposition 4.2, $B^{\prime}$ cannot be incompressible. Since $B^{\prime}$ fully carries $\lambda$ and $B$ contains no disks of contact, $B^{\prime}$ must contain a disk of contact whose boundary must lie in $\nu^{\prime}$, ie, there must be a disk in $N\left(B^{\prime}\right)$ transverse to the $I$-fibers and with boundary in $\nu^{\prime}$. By our construction of $B^{\prime}$ and $\nu^{\prime}$, there must be a splitting annulus $A$ in $N(B)$ connecting $\nu$ and another component of $\partial_{v} N(B)$ with $A \cap \lambda=\emptyset$ and $A \cap R$ a nontrivial arc in $A$.

Intuitively, if $M$ is atoroidal, one should be able to eliminate all tori carried by a branched surface in finitely many steps of splitting as in Lemma 4.1. However, the situation can be very complicated if the branched surface carries infinitely many tori. These tori can tangle together in a complicated way, and it is not clear to us whether there is a simple way to deal with it. This problem can be simplified to a great extent, if we use a special kind of triangulation, namely the one-efficient triangulation due to Jaco and Rubinstein [25]. A triangulation is one-efficient if every normal torus is either thin or thick edge linking. A trivial consequence of using a one-efficient triangulation is that there are only finitely many normal tori, which is the only thing we need for our purpose. 
Lemma 4.4 Suppose $M$ is an atoroidal 3-manifold with a one-efficient triangulation $\tau$. Then there is an algorithm to construct finitely many incompressible Reebless branched surfaces such that every (nowhere dense) essential lamination normal with respect to $\tau$ is fully carried by one of these branched surfaces.

Proof As before, we start with finitely many normal branched surfaces. Since $\tau$ is one-efficient, any normal 2 -sphere is normal isotopic to the vertex linking 2 -sphere. If a normal branched surface $B$ carried a 2 -sphere, then some component of $\partial_{h} N(B)$ would be a 2-sphere parallel to the vertex linking 2-sphere, so $B$ could not fully carry an essential lamination, since then it would have a sphere leaf. So we may assume $B$ carries no 2-spheres. We eliminate all disks of contact, then take sub-branched surfaces. Since there are only finitely many normal tori, by Lemma 4.1, we can split these branched surfaces in finitely many steps to construct finitely many branched surfaces that do not carry any tori, and each normal essential lamination is carried by one of them, then we get rid of disks of contact again. So, these branched surfaces contain no Reeb components. By taking sub branched surfaces if necessary, we can assume each normal essential lamination is fully carried by one of finitely many such branched surfaces.

Let $B$ be a branched surface constructed above. $B$ contains no disk of contact and does not carry any torus. Suppose $B$ fully carries an essential lamination $\lambda$ but $M-B$ has a monogon. By Proposition 4.3, there is a splitting annulus $A(A \cap \lambda=\emptyset)$ connecting two components $v_{1}$ and $v_{2}$ of $\partial_{v} N(B)$. Next, we analyze annuli carried by $N(B)$ with exactly two boundary circles lying in $v_{1}$ and $v_{2}$ respectively by solving a system of branch equations as in [13]. Note that we are considering surfaces with boundary, so some equations are like $x_{i}+x_{j}=x_{k}-1$ and this system of linear equations is not homogeneous. As in [13], there is a one-to-one correspondence between non-negative integer solutions to this system of branch equations and surfaces carried by $B$ with exact two boundary circles lying in $v_{1}$ and $v_{2}$ respectively. Such a solution is a point in $\mathbb{R}^{m}$ with non-negative integer coordinates, where $m$ is the number of variables.

Suppose $A_{1}=\left(a_{1}, \ldots, a_{m}\right)$ and $A_{2}=\left(b_{1}, \ldots, b_{m}\right)$ are two solutions to the system above, and suppose $\chi\left(A_{1}\right)=\chi\left(A_{2}\right)=0$, where $\chi\left(A_{j}\right)$ denotes the Euler characteristic of the surface $A_{j}$. If $a_{i} \leq b_{i}$ for each $i$, then $T=$ $\left(b_{1}-a_{1}, \ldots, b_{m}-a_{m}\right)$ is a non-negative integer solution to the corresponding homogeneous system, and hence $T$ is a union of closed surfaces carried by $B$. Moreover, $A_{1}+T=A_{2}$ implies $\chi\left(A_{1}\right)+\chi(T)=\chi\left(A_{2}\right)$. Hence, $\chi(T)=0$. 
Since $B$ does not carry any 2 -sphere or torus, this is impossible. So, there are no such pairs of annuli among integer solutions. Now, we interpret zero Euler characteristic as a linear equation and add this equation to the system above. Then, every non-negative integer solution to this new system gives us an annulus (or a pair of Möbius bands). Since there are no two annuli $A_{1}$ and $A_{2}$ as above, there are only finitely many non-negative integer solutions to this new system, and hence there are only finitely many possible splitting annuli connecting $v_{1}$ and $v_{2}$. Note that since this is a system of linear equations with integer coefficients, the solution space must be bounded in the region of $x_{i} \geq 0$ (for all $i$ ), otherwise there would be infinitely many non-negative integer solutions. Thus, one can calculate the maximum for each coordinate, and list all non-negative integer solutions, ie, one can list all possible splitting annuli.

After splitting along these splitting annuli, we can eliminate all monogons and get finitely many branched surfaces. By taking sub branched surfaces if necessary, we have that, after isotopy, each normal essential lamination is fully carried by one of these branched surfaces.

Finally, for each of these branched surfaces, we subdivide the triangulation so that the branched surface lies in the 2-skeleton, and then using the algorithm described in lemma 2.9 to check that each branched surface is incompressible and Reebless.

Remark 4.5 (1) If $M$ is a small Seifert fiber space, one can always recognize this manifold if it does not admit a one-vertex triangulation [25]. Moreover, using layered solid tori, one can construct a nice triangulation for a small Seifert fiber space [23] that also makes the proof work, though it may not be one-efficient.

(2) One does not need a one-efficient triangulation to eliminate all Reeb branched surfaces. Suppose $T$ is a torus carried by $N(B)$ and bounding a solid torus $V$, and $V \cap N(B)$ fully carries a sublamination of a Reeb foliation of a solid torus. Then, each $I$-fiber of $N(B)$ can intersect $T$ in at most 2 points. Otherwise, since $T$ is separating, if an $I$-fiber intersects $T$ in more than 2 points, there must be a subarc of this $I$-fiber properly embedded in the solid torus $V$. This contradicts a well-known fact that there is no properly embedded compact arc in a solid torus that is transverse to the Reeb foliation (extended from the Reeb lamination). Therefore, $B$ contains only finitely many Reeb components.

(3) By replacing a leaf of a lamination by an $I$-bundle over this leaf and then deleting the interior of this $I$-bundle, one can change every lamination 
to a nowhere dense one (see Remark 4.4, [21]). Gabai proved that, up to isotopy, every nowhere dense essential lamination in an atoroidal 3manifold is fully carried by one of finitely many essential branched surfaces (Theorem 6.5 of [19]). However, step 1 of the proof of Theorem 6.5 of [19] uses Plante's argument on the limit of normal annuli, and it does not clearly give an algorithm to find these finitely many essential branched surfaces. In particular, if a branched surface carries infinitely many tori, the picture of those normal annuli can be very complicated, and it is not clear how to algorithmically analyze the limit of these annuli.

Theorem 4.6 There is an algorithm to decide whether a 3-manifold contains an essential lamination.

Proof The first step of the algorithm is to modify the triangulation of $M$ to a one-efficient triangulation $\tau$ using the algorithm in [25]. By [25], in finitely many steps, we either get a one-efficient triangulation, or $M$ contains an incompressible torus, or we can recognize $M$ as certain small Seifert fiber space. An incompressible torus can be found, if one exists, by [24] or by Algorithm 8.2 of [26], and essential laminations in small Seifert fiber spaces are classified by $[1,11,27,30]$. So, we know whether $M$ is laminar in these exceptional cases, and we can assume our triangulation for $M$ is one-efficient.

By Lemma 4.4, we can algorithmically construct finitely many incompressible Reebless branched surfaces such that every nowhere dense essential lamination normal with respect to $\tau$ is fully carried by one of them. Then, if one of these incompressible Reebless branched surfaces fully carries a lamination (hence it is an essential lamination), the procedure described in theorem 2.8 will stop, which means $M$ is laminar. If none of them fully carries a lamination, the procedure described in theorem 3.6 will stop, which means $M$ contains no essential lamination.

\section{$5 \quad$ Recognizing Reebless foliations}

In this section, we will assume the reader is familiar with the notion of a sutured manifold, introduced by Gabai in [15]. We will follow the notation of [15].

Remark We note that if a manifold $M$ is atoroidal and admits a Reebless foliation, then the foliation is taut. But in the case of irreducible toroidal manifolds, there are examples that admit Reebless foliations, but no taut foliation 
[5]. It seems to be unknown in general when a toroidal manifold admits a taut foliation. Thus, in the theorems below, we will restrict ourselves to considering Reebless foliations.

Theorem 5.1 Given a sutured manifold $(M, \gamma)$ with triangulation $\tau$ and $\gamma \subset \tau^{(1)}$, there is an algorithm to determine if $(M, \gamma)$ is taut.

Proof By theorem 4.2 of [15], a connected taut sutured manifold $(M, \gamma)$ has a sutured manifold hierarchy

$$
\left(M_{0}, \gamma_{0}\right) \stackrel{S_{1}}{\longrightarrow}\left(M_{1}, \gamma_{1}\right) \stackrel{S_{2}}{\longrightarrow} \cdots \stackrel{S_{n}}{\longrightarrow}\left(M_{n}, \gamma_{n}\right)
$$

such that $\left(M_{n}, A\left(\gamma_{n}\right)\right)=(R \times I, \partial R \times I)$ and $R_{+}\left(\gamma_{n}\right)=R \times 1$. Moreover, for each component $V$ of $R\left(\gamma_{i}\right), S_{i+1} \cap V$ is a union of $k(\geq 0)$ parallel oriented non-separating simple closed curves or arcs. Moreover, if $(M, \gamma)$ has a sutured manifold hierarchy such that no component of $R\left(\gamma_{i}\right)$ is a compressible torus, then $(M, \gamma)$ is taut by corollary 5.3 of $[15]$.

The algorithm to determine if $(M, \gamma)$ is taut proceeds by running two procedures. The first procedure constructs subdivisions $\nu$ of the triangulation $\tau$ searching for surfaces $(S, \partial S) \subset(M, \gamma) \cap \nu^{(2)}$ such that $[S, \partial S]=\left[R_{+}, \gamma\right] \in$ $H_{2}(M, \gamma)$, and $x(S)<x\left(R_{ \pm}\right)$. If $(M, \gamma)$ is not taut, then this procedure terminates in a finite number of steps.

The second algorithm searches for a sutured manifold hierarchy. Again, it constructs subdivisions $\nu$ of $\tau$, and searches for oriented surfaces $S_{i} \subset \nu^{(2)}$ such that they satisfy the conditions for a sutured manifold hierarchy. By Algorithm 9.7 of [26], there is an algorithm to check that $\left(M_{n}, A\left(\gamma_{n}\right)\right) \cong(R \times I, \partial R \times I)$. There are also algorithms to check the other conditions listed above. By Gabai's theorem, if $(M, \gamma)$ is taut, then this procedure will halt in finitely many steps by finding a sutured manifold hierarchy which certifies that $(M, \gamma)$ is taut.

Theorem 5.2 There is an algorithm to decide whether an orientable 3-manifold contains a Reebless foliation.

Proof First, we describe the algorithm.

Step 1 We check that $M$ is irreducible using an algorithm of Rubinstein [34] and Thompson [36]. If it is not irreducible, then we check to see if $M$ is $S^{2} \times S^{1}$, by cutting along a non-separating sphere, cap off the resulting boundary with balls, and check if we have a ball again. Otherwise $M$ is not prime, and $M$ cannot have a Reebless foliation. If $M$ is irreducible then we continue. 
Step 2 We check to see if $M$ is toroidal. If $M$ is toroidal and irreducible, then it has a transversely orientable Reebless foliation by the method of Corollary 6.5 of $[15]$.

Step 3 If $M$ is irreducible and atoroidal, then we construct finitely many normal essential branched surfaces $B$ which fully carry every normal essential lamination using Lemma 4.4. We check to see if $B$ carries a lamination. We triangulate the complementary regions of $B$. Then we use algorithm 8.2 of [26] to find the maximal $I$-bundle $\left(C, \partial_{v} C\right) \subset\left(M-\operatorname{int} N(B), \partial_{v} N(B)\right)$. We then delete from $M-\operatorname{int} N(B)$ unions of components $I$-bundle components $C^{\prime} \subset C$ in all $2^{\beta_{0}(C)}$ possible ways. We use the algorithm described in theorem 5.1 to determine if the regions of $M-\operatorname{int}\left(N(B) \cup C^{\prime}\right)$ are taut sutured manifolds. If all of them are, then by a theorem of Gabai [15], $M-\operatorname{int}\left(N(B) \cup C^{\prime}\right)$ has foliations transverse to $\partial_{v}\left(N(B) \cup C^{\prime}\right)$, with $\partial_{h} N(B)-C^{\prime}$ as leaves. We may extend these foliations and the lamination carried by the branched surface to a Reebless foliation, using the method of the proof of theorem 5.1, pages 471-477 in [15] (see also constructions 4.16 and 4.17, pages 498-500 of [16]), since $B$ contains no disk of contact.

To see that the algorithm works, we need to show that if $M$ has a Reebless foliation, then for the branched surfaces constructed in Lemma 4.4, one of the essential branched surfaces union some $I$-bundles has as complementary regions taut sutured manifolds which may be extended to a foliation. If $M$ has a Reebless foliation, then by theorem 4.4 of [19], there exists a normal essential lamination such that each complementary region of the normal essential branched surface $B^{\prime}$ carrying this lamination is either a taut sutured manifold or an $I$-bundle, since the normal lamination is obtained from the foliation by first splitting, then evacuating a taut sutured manifold. Moreover, these regions are $\pi_{1}$-injective in the 3-manifold group, by condition (ii) of definition 4.2 [19]. The normal essential lamination fully carried by $B^{\prime}$ is also fully carried by one of the branched surfaces $B$ constructed in Lemma 4.4, and thus there is an essential normal branched surface $B_{0}$ that is a splitting of both $B$ and $B^{\prime}$. So, there is a union of product regions $\mathcal{C}$ such that $N\left(B_{0}\right) \cup \mathcal{C}=N(B)$. Let $\lambda$ be the normal essential lamination fully carried by $B_{0}, B$ and $B^{\prime}$, and suppose $\lambda$ extends to a Reebless foliation $\mathcal{F}$. For any $I$-bundle $E \in \mathcal{C}$, we suppose that the horizontal boundary of $E$ lies in $\lambda$ and the vertical boundary of $E$ consists of essential annuli in $M-N\left(B_{0}\right)$. Moreover, since $B_{0}$ is a splitting of $B$ and $B$ contains no splitting annulus as in the construction in Lemma 4.4, we also assume that $E$ is not an annulus $\times I$ or an $I$-bundle over a Möbius band. Next, we show that after isotopies, $\mathcal{F}$ is transverse to the $I$-fibers of $E$ for any $E$. Note that the case that some vertical boundary component of 
$E$ is not an essential annulus (ie, bounds a $D^{2} \times I$ region) and the case that $E$ is an $I$-bundle over an annulus or a Möbius band with a vertical boundary component in $\partial_{v} N\left(B_{0}\right)$ are easy to prove by similar arguments.

We can split $\mathcal{F}$ into a lamination $\mathcal{F}^{\prime}$ so that $\lambda$ is a sublamination of $\mathcal{F}^{\prime}$ and, after isotopy, $\mathcal{F}^{\prime} \cap E$ is an essential lamination in $E$. By our assumption that $E$ is not an $I$-bundle over an annulus or a Möbius band, the double of $E$ is an $I$-bundle over a closed surface of genus greater than 1. A theorem of Brittenham [3] says that any essential lamination in an $I$-bundle over a closed surface with genus $>1$, containing the boundary as leaves, can be isotoped to be transverse to the $I$-fibers. Thus, we can assume $\mathcal{F}^{\prime} \cap E$ is transverse to the $I$-fibers of $E$. Let $N\left(B_{0}\right) \cup E=N\left(B_{1}\right)$ be a fibered neighborhood of another essential branched surface $B_{1}$. Since the vertical boundary of $E$ consists of essential annuli and $E$ is not an $I$-bundle over an annulus or a Möbius band, $B_{1}$ contains no splitting annulus that cuts through $E$. Note that by the construction in [19], every $I$-fiber of $N\left(B^{\prime}\right)$ (and hence $N\left(B_{0}\right)$ ) is transverse to the foliation $\mathcal{F}$. If there is an $I$-fiber of $E$ that cannot be transverse to the foliation $\mathcal{F}$, then there must be an arc $\alpha$ in a leaf of $\mathcal{F}^{\prime}$ and a subarc $\beta$ of an $I$-fiber in $\partial E$ such that $\alpha \cup \beta$ bounds a monogon disk in $M-\mathcal{F}^{\prime}$. Similar to the proofs of Propositions 4.2 and 4.3 , such a monogon disk implies that $N\left(B_{1}\right)$ contains a splitting annulus cutting through $E$, which gives a contradiction. Hence, after isotopies, the foliation $\mathcal{F}$ is transvere to the $I$-fibers of $E$ for any $E \in \mathcal{C}$.

Therefore, by our assumptions on $B^{\prime}, \lambda$ and $\mathcal{F}$, there must be a union of product regions $C^{\prime}$ in $M-\operatorname{int}(N(B))$ such that $M-\operatorname{int}\left(N(B) \cup C^{\prime}\right)$ consists of taut sutured manifolds. Conversely, if we find such a $C^{\prime}$ for $B$, we can conclude that the essential lamination fully carried by $B$ extends to a Reebless foliation by $[15,16]$ because the complementary regions of $B$ are essential. Thus, the algorithm will succeed if and only if the manifold has a Reebless foliation.

\section{Conclusion}

The algorithm we have described is unsatisfying, since the proof that the algorithm terminates doesn't give us any idea of how long the algorithm will take, and it seems that it would be nearly impossible to implement on a computer. It would be interesting to get an upper bound on how much one needs to split an essential branched surface to get a laminar branched surface. If the bound were good enough, then one might be able to answer the following question. 
Question Is there an NP algorithm to determine if a 3-manifold is laminar?

Other questions of interest:

(1) Is there a finite collection of laminar branched surfaces in $M$ which carry every essential lamination, and if so, is there an algorithm to find them?

(2) Are there algorithms to determine if a manifold has a tight lamination [2], an $\mathbb{R}$-covered foliation [8], a slithering [37], or a pseudo-Anosov flow [14] (these references give definitions of these objects, not necessarily original sources for the definitions)?

(3) Is there an algorithm to determine if the fundamental group of a 3manifold has a circular ordering? If an atoroidal manifold $M$ has a taut transversely orientable foliation, then it has been shown by Bill Thurston that $\pi_{1}(M)$ has a circular ordering, also proven by Calegari and Dunfield in Theorem 6.2 of [9].

(4) Is there an algorithm to find all essential laminations in Dehn fillings on a link? What we have in mind here would be to describe a collection of branched surfaces in a link complement, and a description for each branched surface of which Dehn fillings on the link have essential laminations which meet the link complement in a lamination carried by that branched surface. This has been done for the figure eight knot complement by Tim Schwider [35].

\section{References}

[1] Mark Brittenham, Essential laminations in Seifert-fibered spaces, Topology 32 (1993) 61-85

[2] Mark Brittenham, Essential laminations and Haken normal form, Pacific J. Math. 168 (1995) 217-234

[3] Mark Brittenham, Essential laminations in I-bundles, Trans. Amer. Math. Soc. 349 (1997) 1463-1485

[4] Mark Brittenham, Essential laminations in Seifert-fibered spaces: boundary behavior, Topology Appl. 95 (1999) 47-62

[5] Mark Brittenham, Ramin Naimi, Rachel Roberts, Graph manifolds and taut foliations, J. Differential Geom. 45 (1997) 446-470

[6] Danny Calegari, Foliations with one-sided branching, to appear in Geom. Ded. arXiv:math.GT/0101026,

[7] Danny Calegari, Promoting Essential Laminations, arXiv:math.GT/0210148 
[8] Danny Calegari, The geometry of $\mathbb{R}$-covered foliations, Geom. Topol. 4 (2000) $457-515$

[9] Danny Calegari, Nathan M Dunfield, Laminations and groups of homeomorphisms of the circle, to appear in Invent. Math. arXiv:math.GT/0203192

[10] Andrew Casson, Douglas Jungreis, Convergence groups and Seifert fibered 3-manifolds, Invent. Math. 118 (1994) 441-456

[11] David Eisenbud, Ulrich Hirsch, Walter Neumann, Transverse foliations of Seifert bundles and self-homeomorphism of the circle, Comment. Math. Helv. 56 (1981) 638-660

[12] Sergio R Fenley, Laminar free hyperbolic 3-manifolds, arXiv:math.GT/0210482

[13] W Floyd, U Oertel, Incompressible surfaces via branched surfaces, Topology 23 (1984) 117-125

[14] David Fried, Fibrations over $S^{1}$ with pseudo-Anosov monodromy, Travaux de Thurston sur les surfaces (1979) 251-266, séminaire Orsay, with an English summary

[15] David Gabai, Foliations and the topology of 3 -manifolds, J. Differential Geom. 18 (1983) 445-503

[16] David Gabai, Foliations and the topology of 3-manifolds. III, J. Differential Geom. 26 (1987) 479-536

[17] David Gabai, Convergence groups are Fuchsian groups, Ann. of Math. (2) 136 (1992) 447-510

[18] David Gabai, Problems in foliations and laminations, from: "Geometric topology (Athens, GA, 1993)", Amer. Math. Soc., Providence, RI (1997) 1-33

[19] David Gabai, Essential laminations and Kneser normal form, J. Differential Geom. 53 (1999) 517-574

[20] David Gabai, William H Kazez, Group negative curvature for 3 -manifolds with genuine laminations, Geom. Topol. 2 (1998) 65-77

[21] David Gabai, Ulrich Oertel, Essential laminations in 3-manifolds, Ann. of Math. 130 (1989) 41-73

[22] Wolfgang Haken, Some results on surfaces in 3-manifolds, from: "Studies in Modern Topology", Math. Assoc. Amer. (distributed by Prentice-Hall, Englewood Cliffs, N.J.) (1968) 39-98

[23] William Jaco, personal communication

[24] William Jaco, Ulrich Oertel, An algorithm to decide if a 3-manifold is a Haken manifold, Topology 23 (1984) 195-209

[25] William Jaco, Hyam Rubinstein, One-efficient triangulations of 3-manifolds, preprint

[26] William Jaco, Jeffrey L Tollefson, Algorithms for the complete decomposition of a closed 3 -manifold, Illinois J. Math. 39 (1995) 358-406 
[27] Mark Jankins, Walter D Neumann, Rotation numbers of products of circle homeomorphisms, Math. Ann. 271 (1985) 381-400

[28] Tao Li, Laminar branched surfaces in 3-manifolds, Geom. Topol. 6 (2002) 153194

[29] John W Morgans, Peter B Shalen, Degenerations of hyperbolic structures. II. Measured laminations in 3-manifolds, Ann. of Math. (2) 127 (1988) 403-456

[30] Ramin Naimi, Foliations transverse to fibers of Seifert manifolds, Comment. Math. Helv. 69 (1994) 155-162

[31] S P Novikov, The topology of foliations, Trudy Moskov. Mat. Obšč. 14 (1965) 248-278

[32] Ulrich Oertel, Affine laminations and their stretch factors, Pacific J. Math. 182 (1998) 303-328

[33] Rachel Roberts, John Shareshian, Melanie Stein, Infinitely many hyperbolic 3-manifolds which contain no Reebless foliation, to appear in J. Amer. Math. Soc. http://www.math.wustl.edu/ ${ }^{\sim}$ roberts/research.html

[34] Joachim H Rubinstein, An algorithm to recognize the 3-sphere, from: "Proceedings of the International Congress of Mathematicians, Vol. 1, 2 (Zürich, 1994)", Birkhäuser, Basel (1995) 601-611

[35] Tim Schwider, The classification of essential laminations in Dehn surgeries on the figure-eight knot, Phd, The University of Michigan (2001), available at www. math.Isa.umich.edu $/{ }^{\sim}$ tschwide/

[36] Abigail Thompson, Thin position and the recognition problem for $S^{3}$, Math. Res. Lett. 1 (1994) 613-630

[37] William $\mathbf{P}$ Thurston, 3-Manifolds, foliations, and circles I, preprint, arXiv:math.GT/9712268

[38] William $\mathbf{P}$ Thurston, On the geometry and dynamics of diffeomorphisms of surfaces, Bull. Amer. Math. Soc. 19 (1988) 417-431 